\title{
Maternidad, paternidad y desigualdad de género: los dilemas de la conciliación ${ }^{2}$
}

\section{Raquel Royo Prieto}

Dpto. de Trabajo Social y Sociología, Facultad de Ciencias Sociales y Humanas, Universidad de Deusto

<raquel.royo@deusto.es>

Gizarte-berdintasunerako traba nabarmenetako bat da sexuaren araberako lanaren banaketak irautea, eta, partikularki, eragina izaten du emakumeen landiskriminazioan, eta erronka eta argitu beharreko gaiak azaltzen dizkie ongizate Estatuei, eredu hartutako 'gizon hornitzailearen' paradigma aurrean. Artikulu honetan kontu hartzen diegu arazo horiei, horrenbeste aipatu izan den laneko eta bizimodu pribatuaren kontziliazioari, eta baita ere horren atzetik dauden genero-desberdintasunei. Hori lortzeko, denbora-erabileraren datuak aztergai hartzen ditugu, eta gure testuinguruko emakumeen herritar-estatusarekiko dituen inplikazioak azaltzen; eta horren ostean, EAEko 52 gurasoen eguneroko bizimoduan sartzen gara berauen politika eta zerbitzu publikoetako bizipen, kezka eta iritzien berri izateko, betiere, bizimodu pribatu eta lanaren orekaz jakiteko.

\section{HITZ-GAKOAK:}

Kontziliazioa, familia-lana, genero-rolak, amatasuna, aitatasuna.
La persistencia de la división sexual del trabajo constituye uno de los escollos fundamentales en el avance hacia una sociedad igualitaria, que incide, particularmente, en la discriminación laboral femenina y plantea nuevos retos e interrogantes a los Estados del bienestar, construidos desde el modelo del 'varón proveedor'. En este artículo nos acercamos a esta problemática, abordando la profusamente mencionada conciliación entre la vida laboral y la vida privada, así como las desigualdades de género que subyacen a ella. Para ello, analizamos los datos sobre usos del tiempo y sus implicaciones para el estatus de ciudadanía de las mujeres en nuestro contexto, para seguidamente sumergirnos en la realidad cotidiana de 52 madres y padres de la CAE, lo que nos ha permitido explorar sus vivencias, sus preocupaciones, y sus opiniones sobre las políticas y los servicios públicos relacionados con el anhelado y cuestionado equilibrio entre la vida privada y laboral.

\section{PALABRAS ClaVe:}

Conciliación, trabajo familiar, roles de género, maternidad, paternidad.

\footnotetext{
${ }^{1}$ Este artículo se basa fundamentalmente en Raquel Royo, Maternidad, paternidad y conciliación en la CAE: ¿es el trabajo familiar un trabajo de mujeres?, Bilbao, Universidad de Deusto [en colaboración con Emakunde], 2011.
} 


\section{Introducción}

En los últimos años, la problemática de la conciliación, vinculada a la preocupación por armonizar el ámbito laboral y familiar, ha adquirido notoriedad pública y mediática en las sociedades occidentales (Macinnes, 2005: 35). Sin embargo, con frecuencia se olvida que la necesidad de armonizar estos dos mundos no es sino "un viejo tema" bajo "un nuevo nombre" (Carrasquer, 2006), ya que siempre ha estado presente en la experiencia vital de las mujeres de clase obrera, cuyo trabajo remunerado constituía un medio fundamental para su supervivencia y la de sus familias.

El interés actual por la conciliación responde a un nuevo escenario marcado por el aumento de la participación laboral femenina -que ahora es masiva y atañe a las mujeres de clase media-, las presiones demográficas vinculadas al proceso de envejecimiento y, particularmente, el efecto de la agenda social europea (Moltó, 2005: 45). De esta forma, la conciliación que se plantea en el marco de las políticas de empleo y de igualdad de oportunidades tiene como trasfondo importantes problemas estructurales asociados a los cambios demográficos, económicos y sociales. El envejecimiento de la población, la caída de la natalidad, el desempleo y la inactividad constituyen "un reto para sostener el modelo social europeo" (Carrasquer y Torns, 2005: 138) y suscitan numerosos interrogantes con relación a la cobertura presente y futura de las necesidades de cuidado.

La 'crisis de los cuidados' subraya precisamente la quiebra del modelo anterior de organización social de los cuidados -el modelo del 'varón proveedor'que suponía una (no siempre lograda, pero sí socialmente prescrita) división nítida del trabajo entre una mujer consagrada al ámbito doméstico-familiar y un hombre dedicado al empleo. Los factores previamente descritos, unidos a otros, como la falta de corresponsabilidad masculina o el predominio de un modelo de ciudad no integrada en los usos del espacio, favorecen "un desbalance entre las necesidades de cuidados y las posibilidades de proporcionarlos" (Pérez Orozco y López Gil, 2011: 24-25).

En este contexto, Tobío sostiene que, más que de conciliación, puede hablarse de contradicción entre un "nuevo mundo laboral" - caracterizado por la incorporación de las mujeres- y un "viejo modelo familiar" -en gran medida vigente todavía, basado en la división del trabajo según el género-. La superposición de estos dos modelos (y no su integración) configura una relación contradictoria, e incluso conflictiva, que marca la cotidianidad y se plasma en los discursos de las mujeres -que encarnan más directamente dicha contradicción- que describen la compatibilización de dichas exigencias como algo “imposible” o “muy idílico” (Tobío, 2005: 11, 19).

Teresa Torns (2005: 16) destaca, además, que el actual concepto de conciliación pretende mediar entre dos ámbitos que no son "equitativos" ni “democráticos" - a pesar de que la armonía y la felicidad de los miembros de la familia aparezcan recurrentemente en los imaginarios míticos de referencia-, ocultando o negando el conflicto derivado de la división sexual del trabajo que, cuando aflora, tiende a verse como un "asunto privado". Estos factores, señala Torns, están en la base de las limitaciones del concepto tal y como se usa en la mayor parte de las políticas sociales y se plasma en la idea de que la conciliación constituye una problemática femenina. En la misma línea, Sonia Parella (2005: 1) sugiere que el riesgo de estas políticas en los términos que actualmente se plantean es hacer "más llevadera la doble presencia con la que cargan las mujeres".

En este artículo, partimos de los datos sobre los usos del tiempo y otras estadísticas que desvelan la desigualdad de género que persiste en el reparto del trabajo familiar, y analizamos sus implicaciones para el estatus de ciudadanía de las mujeres. Pero, además, tratamos de aproximarnos a la conciliación de la vida personal, familiar y laboral desde la perspectiva de sus indiscutibles protagonistas. Para ello, nos hemos acercado a la realidad cotidiana de veintiséis hogares de estrato social medio de la Comunidad Autónoma de Euskadi (CAE) con distribuciones dispares del trabajo reproductivo y productivo ${ }^{2}$. En las páginas siguientes, nos sumergiremos en sus mundos cotidianos y exploraremos sus prácticas, sus preocupaciones y sus vivencias en este ámbito.

\section{Género y usos del tiempo: las cifras de la desigualdad}

Según los datos de la Encuesta Europea de Valores en su aplicación a la CAE, una abrumadora mayoría de sociedad vasca (96,5\%), en consonancia con el entorno estatal (92,8\%), sostiene que "los hombres deberían asumir tantas responsabilidades como las mujeres en el hogar y con los hijos" (Royo, 2012: 42). En pocas preguntas de la encuesta encontraremos tanta unanimidad entre la población. Este ideal de corresponsabilidad en el trabajo reproductivo ha pasado así a formar parte del imaginario social en nuestro entorno, cuestionando los referentes propios de la familia tradicional, que atribuyen a las mujeres las tareas domésticas y de cuidado.

Sin embargo, esta exaltación de la igualdad y del papel activo de los hombres en el ámbito doméstico-familiar contrasta con las prácticas reales que tienen lugar en los hogares, tal y como muestran las estadísticas sobre usos del tiempo, que han demostrado ser un instrumento valioso para conocer cómo se organizan las sociedades (Eustat, 2006:

\footnotetext{
2 Se trata de 52 personas con estudios medios o superiores, que integran hogares de doble ingreso y que residen con su pareja heterosexual y su prole en alguna localidad de ámbito urbano de la CAE. Hemos denominado 'parejas simétricas' a aquellas en las que los miembros de la pareja consideran que el reparto del trabajo familiar es igualitario, según su definición subjetiva. Viceversa con las 'parejas asimétricas'.
} 
87), visibilizando la forma en que mujeres y hombres tejen la vida social con sus actividades, trabajos -remunerados o no-, descansos y aficiones. Estos estudios han establecido con claridad que, a pesar de la incorporación masiva de las mujeres al empleo, a los diversos niveles educativos y a la arena política, persisten diferencias significativas según el género en la dedicación a las tareas domésticas y al cuidado, al trabajo remunerado y al ocio (INE, 2011, 2004; Emakunde, 2011; Durán, 2010; Instituto de la Mujer, 2007; Aliaga, 2006; Eustat: 2006).

Los datos aportados por la Encuesta de Empleo del Tiempo, desarrollada por primera vez por el Instituto Nacional de Estadística (INE) para todo el territorio estatal en el periodo 2002-2003 y aplicada de nuevo en 2009-2010, se sitúan en esta línea, evidenciando la persistencia de una división especializada de roles, en virtud de la cual las mujeres asumen mayoritariamente el trabajo reproductivo, mientras que los hombres afrontan en su mayoría el remunerado (INE, 2004: 369-370; INE, 2011). Según los últimos datos disponibles, la población femenina de 10 y más años emplea diariamente más de dos horas que los hombres de la misma edad en el trabajo doméstico y el cuidado ( 4 h 7 min frente a 1 h 54 min), mientras que ellos dedican en torno a una hora más que ellas al empleo ( $3 \mathrm{~h} 4 \mathrm{~min}$ frente $\mathrm{a} 1 \mathrm{~h} 54 \mathrm{~min}$ ), lo que redunda en un disfrute más reducido del tiempo de libre por parte de las mujeres, casi una hora menos. Esta diferencia afecta a todas las actividades de ocio, de forma que las féminas dedican diariamente a la vida social y diversión 3 minutos menos que los hombres, practican deporte 15 minutos menos, cultivan sus aficiones y se dedican a Internet 20 minutos menos, y acceden a los medios de comunicación (prensa, radio, televisión) 13 minutos menos (INE, 2011).

En la CAE, la Encuesta de Presupuestos de Tiempo, en la línea de lo afirmado para el conjunto de la población española, confirma la mayor dedicación de las mujeres a los trabajos domésticos y de cuidados, y su menor acceso a las actividades relacionadas con el ocio, tal y como se observa en la Tabla 1. Las investigaciones muestran, por tanto, que a pesar de que se ha producido una importante modificación de valores en el plano consciente, ésta aún se plasma escasamente en las prácticas cotidianas del conjunto de la población.

Tabla 1. Tiempo medio social por tipo de actividad, según sexo. CAE, 2008 (hh:mm)

\begin{tabular}{l|c|c}
\hline Actividad & Mujeres & Hombres \\
\hline Necesidades fisiológicas & $11: 38$ & $11: 43$ \\
\hline Trabajo y formación & $2: 49$ & $4: 00$ \\
\hline Trabajos domésticos & $3: 25$ & $1: 19$ \\
\hline Cuidados a personas del hogar & $0: 36$ & $0: 19$ \\
\hline Vida social & $0: 36$ & $0: 41$ \\
\hline Ocio activo y deportes & $1: 05$ & $1: 38$ \\
\hline Ocio pasivo & $2: 48$ & $3: 10$ \\
\hline Trayectos & $1: 03$ & $1: 09$ \\
\hline
\end{tabular}

Fuente: Elaboración propia a partir de la Encuesta de Presupuestos de Tiempo 2008 (Eustat, 2011).
Cabe matizar que, aunque los colectivos de mujeres y hombres no son entidades homogéneas y sus usos del tiempo dependen de la incidencia de diversas variables, los hombres dedican menos tiempo que las mujeres al hogar y a la familia considerando cada grupo de edad, estado civil, clase socioeconómica, edad o nivel de estudios (Meil, 2005) 3 . Por tanto -y a pesar de la heterogeneidad que, de hecho, existe en el colectivo masculino y, particularmente, en el femenino-, tal y como señala Izquierdo, “la cuestión sobre quién ahorra su tiempo en reproducción y quién lo gasta es bastante obvia". Esta desigualdad entre mujeres y varones en el uso del tiempo tiene profundas implicaciones, ya que, como continúa la autora, "esas horas de más dedicadas a limpiar, cocinar, fregar, etc., son horas que se ahorra el compañero, los hijos e hijas y que pueden dedicar a sí mismos, a su propio crecimiento y mejora personal" (Izquierdo, Del Río y Rodríguez, 1988: 29, 33).

El panorama aún asimétrico que se refleja en los datos, y que se caracteriza -como venimos describiendo- por un mayor tiempo de ocio masculino y una mayor carga global de trabajo femenina, viene acompañado de algunos indicios de transformación del modelo diferencial de distribución de actividades en función del género en el ámbito estatal (INE, 2011; Aliaga, 2006) y en la CAE, según se desprende del análisis comparativo de las encuestas de 1993, 1998, 2003 y 2008 (Emakunde, 2011; Eustat, 2006). En este sentido, y desde una perspectiva diacrónica, se han producido ciertas transformaciones en el ejercicio de la paternidad y en el reparto del trabajo familiar (Institut d'Estudis Regionals i Metropolitans de Barcelona, 2006; Eustat, 2006; Valiente, 2005; Meil, 2005; Alberdi, 1999). Estamos ante lo que Meil califica como "lento pero cierto cambio" en la definición de las pautas tradicionales de división del trabajo doméstico (Meil, 1999: 111), al menos dentro de las "nuevas familias urbanas" (más jóvenes, con mayores niveles educativos y portadoras de valores más igualitarios) [Meil, 1997: 70].

Sin embargo, como hemos expuesto, y a pesar de los cambios en este ámbito, los estudios sobre usos del tiempo constatan la persistencia de diferencias sustanciales según el género en el modo de organizar el tiempo diario en las sociedades española y vasca -como en numerosos países europeos-. Son las cifras de la desigualdad, que sugieren importantes inequidades en la calidad de vida y en la autonomía personal de mujeres y hombres.

${ }^{3}$ El desempeño de un empleo por las mujeres suele suponer una reducción en su participación doméstica (Eustat, 2006: 96; Meil, 2005: 21) y un incremento de la del varón (Durán, 1988: 276). Sin embargo, cuando el hombre está desempleado no se invierten los roles, es decir, "el hombre en casa" no se convierte en "amo de casa" (Meil, 2006: 27, 28), y su participación en las tareas sólo es apreciablemente mayor en las edades que coinciden con la crianza de la prole (si bien también aumenta con la jubilación) [Meil, 2005a: 21]. 


\section{Conciliación, ciudadanía y corresponsabilidad}

En su Crítica política de la economía doméstica, Ma Ángeles Durán señala que uno de los argumentos más empleados para "restar valor" y para ignorar la condición económica del trabajo familiar es "negarle su carácter de trabajo” (Durán, 1978: 23), reservando tal consideración para aquel que se desarrolla en la esfera pública y por el que se obtiene una remuneración. Invisibilizado, naturalizado y devaluado, el trabajo doméstico y familiar -y, por ende, la esfera doméstica- se concibe, en el pensamiento científico y en las creencias socialmente compartidas, como un ámbito totalmente desvinculado de la esfera productiva.

Sin embargo, el trabajo familiar es una condición imprescindible para que el mercado exista. El trabajo reproductivo crea las condiciones necesarias para posteriormente adquirir, entre otras cosas, el capital humano. Pero, sobre todo, este trabajo es determinante para que los seres humanos crezcamos y nos desarrollemos como personas sociales, con habilidades relacionales y seguridades afectivas, en definitiva, con "todas aquellas características que nos convierten en personas" (Grupo Dones i Treballs, 2003: 18). Por ello, $M$ ạ Ángeles Durán señala:

Para la mayoría de las mujeres, su jornada es su vida y su vida, su jornada. Si ellas dejasen, como nuevas versiones de atlantes, de producir, sostener y mantener el mundo, este mundo hecho de cuidados y nuevas vidas se desmoronaría (Durán, 1986: 33).

Pero, siendo el trabajo reproductivo tan importante para las personas individuales y para el conjunto de la sociedad, es decir, para el sostenimiento de la vida humana, ¿cómo es posible que las actividades que lo conforman hayan sido tradicionalmente invisibilizadas y carentes de reconocimiento social y político? Carrasco (2003: 33-34), reconociendo la complejidad de la cuestión, apunta dos grandes razones. La primera se refiere a "las razones del patriarcado". Tal y como señala esta autora, en cualquier sociedad, el grupo dominante impone su concepción del mundo y sus valores, presentándolos como universales. Así, bajo el patriarcado sólo se ha valorado socialmente aquello que guarda relación con la actividad tradicionalmente desempeñada por los hombres -es decir, el empleo-y se ha infravalorado el trabajo familiar, predominantemente realizado por mujeres. La segunda razón reside en el funcionamiento de los sistemas económicos. Éstos, aun presentándose como autónomos, siempre han dependido de una actividad doméstica que reproduce la vida y la fuerza de trabajo sin coste alguno para dichos sistemas. En particular, en el capitalismo esta relación permanece oculta para asegurar "el desplazamiento de costes desde la producción capitalista hacia la esfera doméstica” (Carrasco, 2003: 34).

El olvido de la esfera reproductiva tiene importantes consecuencias en el diseño de las políticas públicas y en la distribución de los beneficios sociales, que no tienen en cuenta que los modos en los que las personas gastan su tiempo son seguramente tan importantes como los modos en los que gastan su dinero, tal y como señalan Folbre y Bittman:

El trabajo de cuidar a las personas dependientes tiene consecuencias positivas para la sociedad en su conjunto, creando y manteniendo a la nueva generación que constituirá la fuerza de trabajo y la ciudadanía. Sin embargo, los individuos [...] que realizan importantes aportaciones a la familia y al trabajo comunitario generalmente pagan una gran multa económica y social (Folbre y Bittman, 2004: 1).

En este sentido, las interacciones entre el espacio doméstico-familiar y el espacio laboral -o la arena política- son fundamentales para comprender cómo la estructura de género construye la desigualdad entre mujeres y hombres en las sociedades patriarcales (Papí y Frau, 2005: 150). Lo que Ingrid Palmer (1992: 79) denomina el 'impuesto reproductivo' de las mujeres -su trabajo no remunerado, gratuito e invisible en el hogar- constituye así un factor fundamental para comprender la desigualdad de las mujeres en el ámbito público, es decir, su precaria o débil inserción en el mercado laboral global, su escasa presencia en las instituciones políticas, su práctica ausencia de los poderes fácticos y su "posición de subordinación en los diferentes espacios sociales en los que se distribuyen y manejan recursos" (Cobo, 2005: 288).

Desde esta perspectiva, la posición de las mujeres en el mercado laboral -en desventaja- no puede comprenderse sin tener en cuenta los condicionantes o determinantes que impone su rol familiar, de la misma forma que la masculina se explica desde la elevada disponibilidad laboral que le confiere su escasa participación doméstica (Tobío, 1998: 21-22). Este fenómeno y, genéricamente, la posición de las mujeres en las democracias occidentales basadas en la igualdad de derechos para ambos sexos muestran que la desigualdad de género cambia continuamente de forma a lo largo del tiempo para perpetuarse. En este sentido, Rosa Cobo (2005) sugiere que, en este momento histórico, la apuesta del patriarcado es que la igualdad formal no se traduzca en una igualdad real.

La feminización de las medidas orientadas a la conciliación ${ }^{4}$, que en su momento despertaron expectativas de avance, se ha convertido en un lastre que contribuye a re-producir el patriarcado, manteniendo a las mujeres -como proclamaba la 'llustración no consecuente'5 - sujetas a sus funciones reproducti-

${ }_{4}$ El análisis del disfrute de las excedencias para el cuidado de hijas e hijos muestra que se trata de medidas fuertemente feminizadas. En 2011, el uso femenino de excedencias orientadas al cuidado de la prole en el marco estatal representó el 95,5\%, mientras que las referidas al cuidado de familiares supusieron el $85,1 \%$, lo que denota una distribución de las responsabilidades reproductivas aún muy desigual. La CAE se sitúa en la línea de lo afirmado para el conjunto del Estado, con porcentajes que ascienden al $94,8 \%$ y $84,1 \%$, respectivamente (Ministerio de Empleo y Seguridad Social, 2011).

El pensamiento ilustrado generalmente negó a las mujeres los derechos que se derivan de la libertad y la igualdad y, por ende, el 
vas, consideradas ahora no únicas para ellas, pero sí prioritarias frente a los nuevos roles laborales femeninos.

Esta feminización se relaciona con condicionantes materiales y socioculturales. Los primeros se refieren a las desigualdades de género existentes en el mercado laboral -acceso a éste, estatus laboral, brecha salarial-, que sitúan a las mujeres en una situación "preferente" para acogerse a medidas de conciliación (Silvestre, 2007), así como a la creciente desincronización de los horarios laborales, acentuada por la falta de servicios asequibles y de calidad, y de prestaciones económicas y otras políticas que faciliten la conciliación (Torns, Borràs y Carrasquer, 2003: 120-121). Los segundos aluden a la pervivencia del modelo del 'varón proveedor' en el imaginario colectivo -que configura a las mujeres como las responsables del trabajo familiar- (Torns, 2005: 24). En dicho modelo, la paternidad se asocia a la función primaria de proveedor familiar y de apoyo emocional para la madre (Barnett y Baruch, 1988: 66), así como a una figura autoritaria y disciplinadora (Moss, 1995: XI), mientras que la imagen de la madre tradicional está marcada por la mitificación del vínculo materno-filial, siendo ella la última responsable del cuidado y del bienestar de la prole, a quien ha de dedicarse en exclusiva (Di Quinzio, 1999). Estos significados culturales de la maternidad y la paternidad están vinculados a las definiciones tradicionales de feminidad y masculinidad, es decir, a lo que se considera socialmente ser hombre o mujer (Coltrane, 1998: 7) ${ }^{6}$. Otros elementos de dicho modelo son el significado devaluado del trabajo doméstico - que se percibe como un no trabajo- y la diferente percepción del tiempo propio (Murillo, 1996), en virtud de la cual los hombres tienen derecho a un tiempo personal, mientras que las mujeres son contempladas como 'seres-para-los-otros'. Este marco favorece que las mujeres actúen como "variable de ajuste" (Grupo Dones i Treballs, 2003: 24) a la hora de armonizar el ámbito familiar y laboral.

En este contexto, se buscan soluciones privadas a la problemática social del cuidado, y son fundamentalmente las mujeres quienes recurren a múltiples estrategias en virtud de su estrato socioeconómico. Las madres con mayores ingresos y nivel educativo pueden recurrir, además de a las redes de apoyo, a la ayuda remunerada que prestan las mujeres de menor nivel socioeconómico, especialmente, las inmigrantes (Badia i Ibáñez, 2003: 17). Esto ha llevado a reflexionar sobre las cadenas de cuidado que se establecen a escala global (Parella, 2000: 275-289; Carrasco, 2003: 47) y que permiten a las mujeres de clase media y alta del Norte construir carreras profe-

acceso al espacio público. Frente a esta Ilustración no consecuente, existen voces que se alzan para reclamar la igualdad para las mujeres, como las de Condorcet, De Gouges y Wollstonecraft, entre otras, que forman parte de la denominada 'Ilustración olvidada' o la 'Ilustración consecuente', que extiende los ideales ilustrados a toda la humanidad (Sánchez Muñoz, 2001: 25).

${ }^{6}$ Aunque la mayor parte de la gente asume que la masculinidad y la feminidad tienen un significado unívoco, lo que es deseable en un hombre o mujer varía significativamente de era en era y de cultura en cultura (Coltrane, 1998: 7) sionales - a las que los hombres siempre han podido acceder a costa de una histórica donación de tiempo femenino-, sin alterar la división sexual del trabajo a escala social, ya que la globalización del cuidado se limita a sustituir a unas mujeres por otras. Este fenómeno evidencia la desigualdad que se establece entre las propias mujeres. Sin embargo, no hay que perder de vista que tanto las mujeres de los países enriquecidos como las de los empobrecidos no son sino "pequeños jugadores en un gran juego económico [y patriarcal, cabría añadir] cuyas reglas no han escrito" (Hochschild, 2003: 189). Además, existe una parte del trabajo familiar que no es delegable, porque no puede trasladarse a los criterios del mercado, y que generalmente continúa recayendo en las féminas occidentales.

Captar las múltiples implicaciones de la desigualdad, los modelos emergentes de cambio -que, como hemos señalado anteriormente, también han aparecido- y, en definitiva, comprender la complejidad del momento actual implica sumergirse en la cotidianidad de los sujetos que encarnan las estadísticas. Las páginas siguientes recogen una aproximación a la realidad particular de las madres y padres que afrontan la necesidad de compaginar dos mundos contrapuestos: familia y empleo. Sus vivencias cotidianas nos acercan a los mecanismos ocultos que contribuyen a reproducir la desigualdad, así como a nuevos valores y conductas que son fundamentales para avanzar hacia una sociedad sin discriminación de género, con un horizonte abierto a maternidades y paternidades más igualitarias.

\section{El lugar de la vida personal}

La conciliación entre empleo y vida privada no se agota en la armonización entre el trabajo productivo y el reproductivo, sino que incluye otras facetas que, sin duda, son imprescindibles para los seres humanos. En este sentido, el debate sobre la conciliación implica aspectos ligados a la idea de vida personal (Chinchilla y Moragas, 2007), como las actividades culturales o lúdicas, cuya exclusión supondría reducir la vida humana al empleo y a la familia (Rivas, 2006). Nuestro acercamiento a la conciliación parte, por tanto, de esta perspectiva integral, que nos permite abordar esta problemática menos explorada.

Para las personas entrevistadas, sus experiencias de maternidad y paternidad suponen -especialmente para las mujeres- una pérdida de importancia de la individualidad en diversos grados. Con frecuencia, los miembros de las parejas entrevistadas manifiestan que el hombre dispone de más tiempo de ocio personal que la mujer, lo que se debe, por una parte, al mayor desempeño de trabajo familiar por parte de ésta, fundamentalmente en los repartos asimétricos. En estos casos, la carga total de trabajo de estas mujeres es generalmente mayor que la de sus cónyuges, aunque dediquen más tiempo que ellas al empleo, lo que se plasma en un mayor tiempo de ocio masculino. Así lo expresa una consultada: 
Yo no hago como él; si tengo cosas que hacer en casa, si hay que hacer la comida, si hay que... lo que sea, cualquier cosa, no me pongo a salir. [...] Él tiene más tiempo libre, aunque trabaje más horas fuera de casa, pero luego, dentro de casa, no mete las mismas horas.

Por otra parte, las mujeres frecuentemente anteponen el tiempo familiar al tiempo personal -tanto en las parejas simétricas como asimétricas-, lo que contribuye a que dispongan de menos tiempo de ocio personal que sus cónyuges. Una de ellas se refiere a este aspecto de la forma siguiente:

Sí, podría dedicar más tiempo al ocio, si quisiera, pero yo a mis hijas no las veo como un trabajo. Desde luego, yo ahora no hago nada de ocio, nada, nada de mi ocio personal, ¿eh? [...] Yo antes hacía mucho deporte y me gustaba mucho; iba a andar en bici también. Yo ahora eso no lo estoy haciendo. Imagínate, tus hijas, tu marido y tú de repente: "Bueno, me voy a andar en bici”. Lo podría hacer, pero no; las estoy viendo jugando y lo que hago es meterme en el grupo.

Los hombres, sin embargo, definen proyectos propios de expansión ajenos a la familia y a la prole, y no se plantean renunciar al tiempo propio por el tiempo familiar. Así se expresa el cónyuge de la entrevistada anterior:

Yo intento sacar más [tiempo de ocio], yo, si por la tarde veo que las niñas están viendo una película o están jugando, cojo y me voy al ordenador. [...] Yo tengo hobbies que hago yo solo.

Estas diferencias en el tiempo de ocio personal podrían ser el resultado de la interiorización de diferentes pautas culturales con relación al uso del tiempo. Mientras que los varones han heredado el derecho a un tiempo propio derivado de su tradicional exención del trabajo familiar, ellas han asumido la domesticidad como mandato genérico que supone anteponer las necesidades y deseos de otras personas a las propias (Murillo, 1996: XXII). Los mandatos de género impelen a las mujeres a convertirse en un 'ser-para-los-otros' -en el centro de sus vidas están las demás personas, no ellas mismas-, mientras que los varones se construyen como 'seres-para-sí-en-elmundo' -se tienen a sí mismos como objetivo de sus energías vitales, como su centro- (Lagarde, 1996: 6o-62). Así, las mujeres no sólo tendrían negado el tiempo privado - por su mayor realización del trabajo familiar-, sino, en buena medida, también el deseo de éste, ya que es considerado como una forma de egoísmo.

El análisis del tiempo libre personal7 es un aspecto fundamental que nos permite aprehender los diversos grados en que las personas consultadas consiguen conciliar su vida personal con sus obligaciones familiares y laborales. Para ello, basándonos en el trabajo empírico realizado, hemos construido cinco modelos o tipos ideales de pareja que describen la forma en que se produce (o no) dicha armonización en parejas de doble ingreso que pertenecen al estrato social medio y que residen en un ámbito urbano de nuestro contexto. Para construir esta tipología, hemos considerado las siguientes variables: el reparto del trabajo reproductivo (simétrico o asimétrico), la distribución del tiempo propio, las concepciones interiorizadas (el mandato cultural de relegar el tiempo propio para las mujeres y el derecho heredado al tiempo propio de los hombres), y la existencia o no de conflicto en la pareja. Podemos representar estos tipos ideales dentro de un continuo que contendría un universo de situaciones en el que virtualmente se podrían ubicar los casos existentes en la realidad.

\section{Figura 1. Tipos ideales de conciliación de la vida personal con la familiar en parejas de doble ingreso}

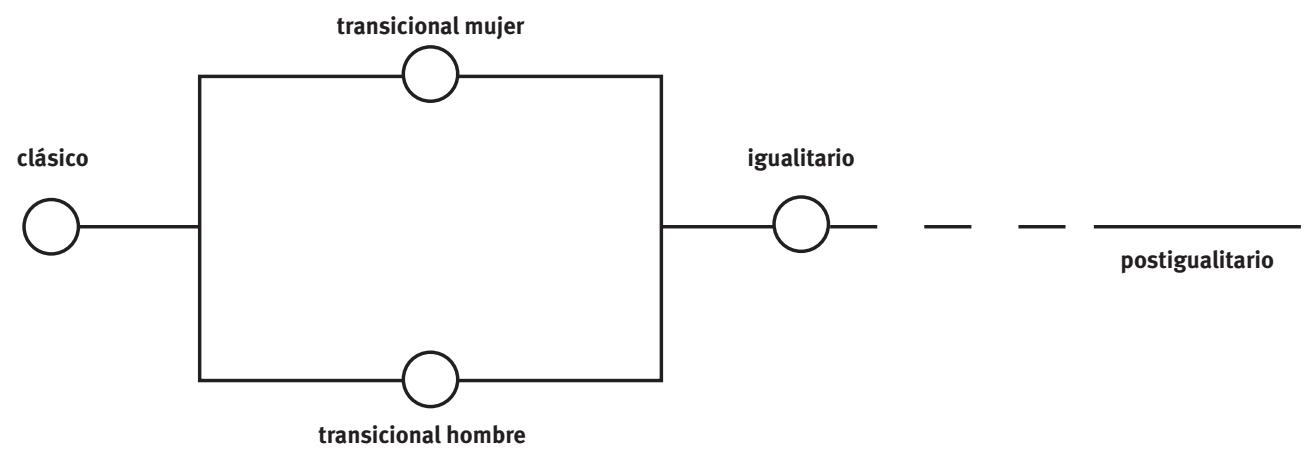

TRADICIÓN

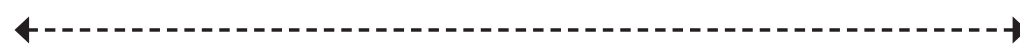

CAMBIO

Fuente: Elaboración propia.

7 El concepto de tiempo libre personal que manejamos en esta investigación procede de la definición subjetiva de las personas entrevistadas. 
El modelo que hemos denominado clásico ocuparía uno de los extremos de dicho continuo. En él, la mujer dispone de menos tiempo propio que el varón, debido a su mayor realización de trabajo familiar y a su interiorización del mandato genérico que prescribe que las mujeres deben anteponer las necesidades de las demás personas a las propias. En este caso, tanto el hombre como la mujer siguen las prescripciones culturales sobre el tiempo propio según el género: él tiene derecho a su tiempo propio al margen de las necesidades familiares y ella renuncia a éste por atender a su familia. En este tipo, ninguno de los dos miembros de la pareja concilia la vida personal y familiar, ya que la mujer desatiende su vida personal, mientras que el varón hace lo mismo con el trabajo familiar. Salvando las distancias, este modelo se aproxima al modelo tradicional, hegemónico en un pasado aún cercano.

Hoy día, nos encontramos en un contexto diferente, sembrado de importantes inercias y también de cambios que transgreden el viejo orden doméstico. Estos últimos se plasman de forma inequívoca en el modelo que hemos denominado igualitario y que consideramos como el tipo más deseable, ya que permite potenciar la conciliación corresponsable. En él, ambos miembros de la pareja consideran que el tiempo propio es un aspecto importante y tratan de dividirlo equitativamente, lo que implica un reparto equitativo del trabajo familiar y el recurso a la negociación. Asimismo, pueden delegar parte del trabajo familiar en una tercera persona ajena a la pareja, pero consideran fundamental dedicar su tiempo al cuidado y educación de la prole. Ambos transgreden los mandatos del género. Este modelo permite que tanto la mujer como el hombre puedan conciliar su vida personal y familiar, de forma igualitaria y sobre la base del acuerdo mutuo.

Pero, además, en un marco social en el que se han expandido los valores igualitarios, resulta plausible que alguna persona del modelo clásico cuestione el reparto del trabajo reproductivo de su hogar, lo que supondría un pequeño movimiento en el continuo desde este modelo hacia los modelos transicionales. Los tipos transicionales encarnan simultáneamente la persistencia y la subversión de conductas y concepciones tradicionales en el seno de la pareja. El tipo transicional con cambio en la mujer implica que la mujer demanda tiempo para sí en un reparto asimétrico, ante un varón que justifica la desigualdad. Esta situación lleva a un conflicto manifiesto en la pareja, ya que con frecuencia esta mujer sólo conseguirá hacer valer su derecho al tiempo propio en la medida en que el varón asuma una mayor responsabilidad familiar. Éste, sin embargo, puede realizar ciertas tareas de forma no habitual, por ejemplo, ante la reiterada solicitud por parte de su pareja o con la intención de reducir el conflicto familiar, manteniendo intactas sus concepciones de género interiorizadas. Aquí se podría iniciar un proceso de transición hacia el modelo igualitario en la medida en que el hombre asumiera más trabajo reproductivo y la mujer, más tiempo propio.
En el tipo transicional con cambio en el varón, la mujer integrante de un reparto simétrico dispone de menos tiempo para sí debido a que lo relega, siguiendo las prescripciones del género. Su compañero, sin embargo, transgrede los mandatos culturales genéricos, al asumir el trabajo familiar de forma equitativa y al animar a la mujer a disponer de un tiempo propio. Él puede acceder a un tiempo propio en diversos grados, aunque la mujer no lo haga. 0 bien puede seguir la pauta conductual femenina y verse forzado a renunciar a su tiempo de ocio personal. En ambos casos, puede existir un conflicto manifiesto en la pareja. En el primero, puede producirse un sentimiento de malestar en el hombre debido al desigual uso del tiempo en la pareja; mientras que, en el segundo, el hombre puede sentir frustración ante la pérdida de un tiempo propio que no está consensuada consigo mismo. En cualquiera de estas situaciones, la mujer no concilia su vida personal y familiar, mientras que el varón puede hacerlo, o no, en diversos grados. En la medida en que la mujer empiece a no postergar su tiempo de ocio personal, estaríamos de nuevo avanzando en el continuo hacia el modelo igualitario.

Estos modelos transicionales ilustran la dinámica del cambio y la complejidad del momento social actual con relación a los valores y conductas familiares. Pero aún podemos referirnos a un último tipo: el postigualitario. Éste se produce en repartos simétricos o cuasisimétricos, y se caracteriza por la reducción de la vida familiar y personal de ambos miembros de la pareja, que recurren a la externalización de una gran parte del trabajo reproductivo (mediante la ayuda remunerada o familiar) para consagrar su tiempo y esfuerzos a su carrera profesional. En el fondo, tanto el varón como la mujer asumen la pauta masculina que prescribe una considerable exención de las tareas domésticas y de cuidado. Al igual que sucedía en el tipo clásico, en este modelo la conciliación de la vida personal y familiar no se produce en ninguno de los miembros de la pareja, debido al menoscabo importante que sufre esta última.

Esta tipología pone en relación las tendencias sociales más amplias -como la incorporación masiva de las mujeres al mercado laboral, la generalmente menor (pero cambiante) participación masculina en el trabajo reproductivo, las ideologías de género, la expansión del principio de igualdad-con los niveles individual e interpersonal. Además, evidencia que, aunque la simetría o asimetría en el reparto del trabajo familiar es un aspecto fundamental en la distribución del tiempo libre entre los miembros de la pareja, la aceptación o el rechazo femenino de los mandatos del género puede constituir un factor decisivo en la configuración del tiempo de ocio personal de las mujeres. En el mismo sentido, las ideas de los varones que justifican la ausencia de tiempo femenino, o tratan de modificarla, también pueden desempeñar un papel relevante. 


\section{Producción y reproducción: ¿ámbitos compatibles?}

La incorporación masiva de las mujeres de clase media al ámbito laboral ha sacado a la luz la oculta(da) relación entre la esfera productiva y reproductiva, y las vidas de nuestras entrevistadas parecen encarnar las interacciones y contradicciones de ambos mundos. Así, la vida familiar -especialmente en las parejas asimétricas- ha supuesto para ellas medidas como la reducción de jornada, el cambio a un empleo de menor estatus, la renuncia a un ascenso o el abandono del puesto de trabajo. A esto se añaden aspectos menos visibles que limitan sus oportunidades en el empleo, como la dificultad para la formación o el 'techo' laboral al que se enfrentan algunas de estas madres. Además, para estas mujeres su trabajo remunerado supone frecuentemente costes emocionales -culpa, sensación de perderse cosas- relacionados con la esfera reproductiva, como relata la siguiente mujer:

Aunque oficialmente haya roto el cordón umbilical con mi hija, no es así. Entonces, hay que empezar a soltar un poco, y yo tengo que empezar a hacer cosas más para mí y también dejar de sentir esa sensación de culpa que tengo por no pasar tanto tiempo con ella, ¿no? Es algo que sí que me martiriza, que me machaca mucho. [...] Yo siento que la abandono. No lo puedo evitar.

Por el contrario, los hombres consultados suelen afirmar que su vida familiar no afecta a su empleo (ninguno de ellos lo ha modificado después de tener descendencia). Asimismo, ningún padre consultado expresa culpa, a pesar de que frecuentemente dedican menos tiempo a sus hijas e hijos que sus compañeras. La ausencia en los entrevistados de las exigentes expectativas culturales hacia las madres (que sí pesan en las mujeres) explica este hecho. En ellos -incluso en los hombres de repartos igualitarios-, el modelo interiorizado es el del 'varón proveedor'.

No obstante, algunos consultados se refieren específicamente a los sentimientos que les suscita el hecho de no poder dedicarse más a la prole, como consecuencia de su empleo, lo cual parece indicar que emergen nuevos modelos de paternidad. Así se expresa el siguiente entrevistado:

Sigo echando de menos eso, la posibilidad de pasar más horas, pasar más horas con la niña. [...] Yo, en mi organización personal y de familia, lo que le he dicho a Nuria es: "El jueves no cojo más pacientes, me dedico a la niña”. Porque es cierto que cada vez vienen más; entonces, eso me pone en un aprieto, porque..., porque sí, es cierto, que ahora, con la casa nueva, se necesita dinero, pero si yo cojo más horas, a ella le cargo más... de estar con la niña y lo que supone eso. Entonces, a mí sí me provoca un conflicto. [...] Sí, es importante mi trabajo, pero también es importante el cuidado de mi hija, y entonces..., de alguna forma..., me encantaría que Nuria pudiera encontrar algo en que estuviera a gusto y yo... ¡uf! ¡Ya está!, ya tengo la excusa perfecta, para decir: "Pues mira, yo, los jueves y viernes, o... lunes, martes y no sé qué, ya [enfatizando] paso de coger a nadie, porque ya estás tú para trabajar y yo me dedico a estar con mi hija".

En este sentido, cabe señalar que la estructura generizada del mercado laboral no sólo se plasma en el mayor trabajo familiar femenino, sino también en las dificultades para ejercer la paternidad, lo que suele pasar más inadvertido para los varones entrevistados $y$, probablemente, para la sociedad en su conjunto. Mientras, las consultadas asumen en su persona el coste de la reproducción social (especialmente en las parejas asimétricas), y las figuras que cuidan de forma vicaria son generalmente femeninas, sin que resulte extraño que los padres sean el 'último recurso’, como relata la siguiente entrevistada:

Aprieto mucho mi agenda, aprieto mucho y puntualmente, si tengo alguna reunión o si tengo alguna tarde que trabajar, pues, se lo pido a la persona que los cuida. Y si ya me parece que es mucho abuso, pues, en un caso extremo, le digo a mi ama. [...] Ahora me apañaría con la que les cuida, y si no, con mi madre; y si no me puedo arreglar, pues Isidro tiene horario flexible y saldría a las tres y también se haría cargo de los chavales.

Sin embargo, podemos referirnos a nuevos roles masculinos que son significativos por la ruptura que suponen con los modelos heredados. En este sentido, podemos mencionar la intervención de algunos varones del entorno social -abuelos y amigos- en el cuidado, el disfrute compartido del permiso de maternidad y el permiso de lactancia de varios consultados, y, especialmente, la participación en el cuidado de los hombres de las parejas simétricas.

En cualquier caso, en los relatos recogidos destacan las múltiples dificultades que las personas consultadas encuentran para armonizar el cuidado de la familia y su desempeño laboral. Sus problemas cotidianos se agudizan con contingencias difíciles de prever, como la necesidad de cuidar, por ejemplo, a una hija enferma, circunstancia que sintetiza muy bien el choque entre la lógica que rige las estructuras productivas y la lógica del ámbito familiar. A diferencia del tiempo destinado a la producción, el tiempo doméstico-familiar exige distintas intensidades a lo largo de la vida y está fuertemente marcado por la imprevisibilidad, lo que dota de complejidad a la problemática de la conciliación, en la medida que ésta supone cambios significativos en el conjunto de la organización sociotemporal (Pérez Sánchez, Cardús y Morral, 2002).

En este contexto de dificultad cotidiana se enmarcan las críticas de estas personas hacia la sociedad, el mercado laboral, el mundo empresarial, la institución escolar, la falta de servicios públicos o, incluso, algunos aspectos de las medidas de conciliación. 
Estas madres y padres señalan que la sociedad y la ciudad no están diseñadas para cuidar y educar, al tiempo que, explícita o implícitamente, sostienen que la reproducción constituye una cuestión pública y política, y no un asunto meramente privado. Así lo expresa uno de los hombres:

La sociedad no está hecha para educar niños. No está hecha. Ni para que los adultos disfruten de los niños. [...] Nos hace estar muy lejos [enfatizando] de cosas que son importantísimas.

Desde una perspectiva comparativa con el entorno europeo, estas personas reclaman horarios 'humanitarios', así como la ampliación de los permisos maternales y paternales, y otras facilidades para la conciliación, tal y como señala una entrevistada:

En otros sitios hay más permisos, en Francia y en Suecia, mucho más. Es que aquí, ¿quién pare y quién cría?, ¿quién tiene hijos?

Ahora bien, al diseñar tales medidas hay que tener en cuenta las discrepancias que se producen entre los derechos legales y las prácticas reales. Las personas que hemos consultado se refieren a la vivencia de inercias institucionales, prejuicios y discriminaciones en sus ámbitos laborales que les impiden ejercer los derechos reconocidos en la ley. A esto se suman los efectos perversos que relatan las mujeres que disponen de reducciones de jornada y de otras medidas de conciliación. La aplicación exclusivamente femenina de estas medidas o la inalterabilidad del 'techo' laboral son algunos de los límites de la conciliación que estas mujeres señalan, basándose en su experiencia:

¡Mira!, en mi oficina hay más tías trabajando que tíos y, sin embargo, los tíos tienen todos los puestos de responsabilidad, y son padres, ¿eh? En el estudio que hicimos [para implementar el plan de igualdad de género en la empresa], hubo hombres que se quedaban flipados, pero esto es porque está así montado, porque ser responsable es estar al cien por cien con un móvil. Si tú eres padre, es como que tu mujer puede dejarlo, ¿no? Aunque seas responsable de un departamento, puedes dejarlo, pero un tío no. Yo tengo casos de parejas trabajando en la misma entidad, hombre y mujer, y reducen ellas y ellos siguen trabajando en su puesto de responsabilidad. [...] Cuando se ponen medidas para la conciliación familiar, pues, deben pensar que están bien para nosotras y que para ellos no existen. Es irrisorio.

Las solicitudes más mencionadas por las personas entrevistadas son la adecuación de los actuales horarios laborales, escolares y comerciales a las demandas del ámbito familiar; el incremento de los servicios y permisos para la conciliación, así como la flexibilidad en el diseño y aplicación de dichas medidas; y la traducción de los derechos legales en su disfrute real. A ello se añade, en ocasiones, la igualdad salarial entre mujeres y hombres. En defi- nitiva, un profundo cambio en la forma de organizar el tiempo y la producción enmarcado en un nuevo modelo social orientado a garantizar la sostenibilidad de la vida, que quizá sea tan complejo como imprescindible.

\section{Más allá de la conciliación: hacia una sociedad corresponsable}

La 'crisis de los cuidados' se produce en un sistema social organizado en torno a la producción, que ha tornado invisible y gratuito un trabajo socialmente imprescindible, como el cuidado. Este sistema social, construido desde el modelo del 'varón proveedor', ha conocido el acceso masivo de las mujeres de clase media al ámbito laboral, sin que los hombres se hayan incorporado al doméstico en una medida equivalente y sin que se haya transformado la organización socioeconómica de forma consecuente con los nuevos roles femeninos. Las últimas décadas han conocido, además, la caída de la natalidad, la crisis de los sistemas de previsión social y la emergencia de la problemática de la conciliación entre la vida personal, familiar y laboral, que se han convertido en problemas sociales de primer orden.

Desde este contexto de cambio y de crisis, se entienden las rupturas que se producen entre los distintos niveles del individuo -consciente, inconsciente, emocional-, así como las disputas maritales en torno al trabajo familiar, a las que nos hemos acercado al abordar los modelos de conciliación de la vida personal con la familiar y laboral en parejas de doble ingreso. Y es que el cambio -social, interpersonal o individual-suele estar acompañado del conflicto. En parte, esto sucede porque el proceso de cambio no es lineal, es decir, no se produce con la misma intensidad en todos los sectores sociales, en todas las personas, ni siquiera en todos los planos de la persona (el cambio en el ámbito familiar es mayor en el plano discursivo y consciente, que en el inconsciente o el conductual). En cualquier caso, las contradicciones del contexto social a las que nos hemos referido son absorbidas especialmente por las mujeres, que asumen en su persona los costos sociales de la conciliación.

La división sexual del trabajo que, como hemos visto, persiste en los hogares perjudica, en particular, a todas aquellas mujeres que pagan el precio de la reproducción social con su falta de tiempo propio, su sentimiento de culpa -emoción que contribuye al manteniendo del status quo y que no hemos encontrado en ningún varón-, o su menoscabo profesional. La desigualdad se reformula y se plasma así en las renuncias personales y laborales unilaterales de las consultadas; en fin, en los múltiples y desiguales costes que el hecho de tener descendencia tiene aún para unas y otros. Pero también los hombres se ven afectados por esta desigualdad que se vuelve contra el sujeto, en principio, privilegiado, cercenando de su personalidad aspectos tan importantes y tan genuinamente humanos como lo emocional, el 
cuidado o la comunicación íntima. Al mismo tiempo, esta discriminación de las féminas en los hogares hace que la sociedad se vea privada de su talento y potencial en el ámbito laboral, así como de las capacidades de los hombres en el reproductivo; toda una pérdida de potencialidades humanas que obstaculiza enormemente el desarrollo individual y colectivo.

Por todo ello, ante la crisis de la estructura socioeconómica, es urgente construir un nuevo modelo social que sitúe en el centro el bienestar social y la sostenibilidad de la vida, lo que supone todo un profundo cambio en la forma de organizar los tiempos y la producción. Una sociedad en la que los diversos agentes sociales -instituciones, empresas, medios de comunicación, escuelas- sean corresponsables, y en la que mujeres y hombres podamos compartir en igualdad las responsabilidades productivas y reproductivas. Para ello, es imprescindible evitar la feminización de las medidas de conciliación, impulsando aquellas que permitan que todas las personas puedan armonizar su vida personal, familiar y laboral, frente a políticas basadas en medidas específicas de conciliación que, debido a los condicionantes culturales y materiales, se enfocan directa o indirectamente hacia las mujeres ${ }^{8}$.
Asimismo, para que realmente se compartan los trabajos y los espacios sociales, es necesario que se produzcan cambios en profundidad en la identidad de mujeres y hombres, transformando los imaginarios míticos de referencia que actúan como invisibles y eficaces obstáculos a la corresponsabilidad. Esto requiere desprendernos de la ideología patriarcal interiorizada que adscribe roles, características, tiempos y espacios diferentes a unas y a otros, deslegitimando 'por 'dentro' y 'por fuera' un sistema que se ha levantado sobre el axioma de la inferioridad de las mujeres y de su subordinación a los hombres (Amorós y De Miguel: 2005). Sólo desde aquí es posible construir modelos igualitarios de conciliación y una sociedad en la que, al contrario de lo que afirma el diagnóstico de Torns, Borràs y Carrasquer (2003: 124), los hombres sí quieran aprender a desempeñar el trabajo familiar y las mujeres sí quieran ceder. Una sociedad que afronte el reto de revalorizar y poner en el centro mismo del sistema una esfera históricamente relegada y construida socialmente como femenina: el cuidado de la vida humana. Sólo en una sociedad así podremos, mujeres y hombres, compartir los trabajos y la vida.

${ }^{8}$ Algunas de las actuaciones en esta línea son la reducción generalizada de la jornada laboral, los permisos de maternidad y paternidad iguales e intransferibles, el fomento de los servicios públicos para el cuidado o la sincronización de horarios laborales y escolares. Para más información sobre este tema, véase Royo (2012). 
(2003) Panorama Sociolaboral de la Mujer en España, [Área de Estudios y Análisis del Consejo Económico y Social], $\mathrm{n}-33$, págs. 1-4.

ALBERDI, I. (dir.) (1999): La nueva familia española, Madrid, Taurus.

ALIAGA, C. (2006): How is the Time of Women and Men Distributed in Europe?, serie: Statistics in Focus, n- 4/2006, Luxemburgo, Comisión Europea [<http://epp.eurostat.ec.europa. eu/portal/page/portal/product_details/ publication?p_product_code=KS-NK-06-004)].

AMORÓS, C.; y DE MIGUEL, A. (eds.) (2005): Teoría feminista: de la llustración a la globalización. De la llustración al segundo sexo, tomo 1, Madrid, Minerva.

BADIA I IBÁÑEZ, M. (2003): "Difficulties ahead for Spanish Families”, Cross-National Research Papers, vol. 6, no 6, págs. 60-66.

BARNETT, R. C.; y BARUCH, G. K. (1988): “Correlates of fathers' participation in family work", en BRONSTEIN, P.; y COWAN, C. P. (eds.), Fatherhood Today. Men's Changing Role in the Family, Nueva York, Wiley, págs. 66-78.

CARRASCO, C. (ed.) (2003): “¿Conciliación? No, gracias. Hacia una nueva organización social”, en AMOROSO, M. I. et al., Malabaristas de la vida. Mujeres, tiempos y trabajos, Barcelona, Icaria, págs. 27-51.

CARRASQUER, P. (2006): “En torno a la conciliación entre la vida laboral y familiar. Una aproximación desde la perspectiva de la doble presencia", Redsi, no 6 [rhttp://redsirevista.cebs-es.org/ documentos/16/93/174.pdf〉].

CARRASQUER, P.; y TORNS, T. (2005): “Tiempos de trabajo: una aproximación desde la conciliación",
Cuadernos de Relaciones Laborales, vol. 23, nํㅜ 1, págs. 9-11.

CHINCHILLA, M. N.; y MORAGAS, M. (2007): Dueños de nuestro destino. Cómo conciliar la vida profesional, familiar y personal, Barcelona, Ariel.

COBO, R. (2005): “Globalización y nuevas servidumbres de las mujeres", en AMORÓS, C.; y DE MIGUEL, A. (eds.), Teoría feminista: de la ilustración a la globalización, tomo 3, Madrid, Minerva, págs. 267-300.

COLTRANE, S. (1998): Gender and Families, serie: The Gender Lens, Thousand Oaks, Pine Fore Press.

DI QUINZIO, P. (1999): The Impossibility of Motherhood: Feminism, Individualism and the Problem of Mothering, Nueva York, Routledge.

DURÁN, M. A. (2010): Tiempo de vida y tiempo de trabajo, España, Fundación BBVA.

- (1986): La jornada interminable, Barcelona, Icaria.

- (1978): El ama de casa. Crítica política de la economía doméstica, Madrid, Zero Zyx.

EMAKUNDE (2011): Cifras sobre la situación de mujeres y hombres en Euskadi 2011 [/http://www. emakunde.euskadi.net/u7220010/es/ contenidos/informacion/cifras_ant/es_ emakunde/adjuntos/cifras_2011_es.pdf)].

EUSTAT (2011): Encuesta de Presupuestos de Tiempo 2008, Vitoria-Gasteiz, Eustat [khttp://www.eustat. es/bancopx/spanish/id_2370/indiceRR.html axzz2N1ryYiwl>].

- (2006): Encuesta de Presupuestos de Tiempo. 2003. Monográficos, Vitoria-Gasteiz, Eustat [<http:// bit.ly/11AkUYq>]. 
FOLBRE, N.; y BITTMAN, M. (eds.) (2004): Family Time. The Social Organization of Care, Padstow, Routledge.

GRUPO DONES I TREBALLS (2003): “Introducción: repasar desde el feminismo los tiempos y trabajos en la vida cotidiana", en AMOROSO, M. I. et al., Malabaristas de la vida. Mujeres, tiempos y trabajos, Barcelona, Icaria, págs. 13-25.

HOCHSCHILD, A. R. (2003): The Commercialization of Intimate Life. Notes from Home and Work Berkeley, University of California Press.

INE (2011): Encuesta de Empleo del Tiempo 2009-2010. Resultados definitivos Madrid, Instituto Nacional de Estadística [<http://www.ine.es/ daco/dac042/empleo/dacoempleo.htm〉].

- (2004): Encuesta de Empleo del Tiempo 2002-2003. Tomo I. Metodología y Resultados Nacionales, Madrid, Instituto Nacional de Estadística.

INSTITUT D'ESTUDIS REGIONALS I METROPOLITANS DE BARCELONA (2006): L'ús social del temps a Barcelona. Informe quantitatiu, Barcelona, Ayuntamiento de Barcelona [<http:// www.iermb.uab.es/htm/publicacions. asp?idPubCat $=8 \&$ idPub $=13\rangle]$

INSTITUTO DE LA MUJER (2007): Usos del tiempo, estereotipos, valores y actitudes, serie: Estudios, nํㅜ 101, Madrid, Instituto de la Mujer.

IZQUIERDO, M. J.; DEL RÍO, O.; y RODRÍGUEZ, A. (1988): La desigualdad de las mujeres en el uso del tiempo, serie: Estudios, Madrid, Ministerio de Asuntos Sociales Instituto de la Mujer.

LAGARDE, M. (1996): Género y feminismo: desarrollo humano y democracia, serie: Cuadernos Inacabados, $\mathrm{n}^{0} \mathbf{2 5}$, Madrid, Horas y Horas.

MACINNES, J. (2005): "Diez mitos sobre la conciliación de la vida laboral y familiar", Cuadernos de Relaciones Laborales, vol. 23, nํㅜ 1, págs. 35-71.

MEIL, G. (2005): El reparto de responsabilidades domésticas en la Comunidad de Madrid, Madrid, Comunidad de Madrid, Consejería de Familia y Asuntos Sociales, Dirección General de Familia.

- (1999): La postmodernización de la familia española, Madrid, Acento.

- (1997): “La redefinición del trabajo doméstico en la nueva familia urbana española", Reis, $n$ 요 80, págs. 69-93.

MOLTÓ, M. L. (2005): “Los recursos para la conciliación entre la vida laboral y familiar: efectos de las políticas de conciliación", Aequalitas. Revista Jurídica de Igualdad de Oportunidades entre Mujeres y Hombres, no 17, págs. 41-46.

MOSS, P. (1995): Father Figures. Fathers in the Families of the 1990s, Edimburgo, Her Majesty's Stationery Office.

MURILLO, S. (1996): El mito de la vida privada: de la entrega al tiempo propio, Madrid, Siglo XXI.

PALMER, I. (1992): "Gender equity and economic efficiency in adjustment of programmes", en AFSHAR, $\mathrm{H}_{\text {.; }} \mathrm{y}$ DENNIS C. (ed.), Women and Adjustment in the Third World, Basingstoke, Macmillan, págs. 69-83.
PAPÍ, N.; y FRAU, M. J. (2005): “La conciliación del empleo y del hogar: respuesta y reflejo de una organización del trabajo construida desde la institución del género", Reis, nํㅜ 110, págs. 149-171.

PARELLA, S. (2005): La conciliación de la vida familiar y laboral a debate. Riesgos y oportunidades desde una perspectiva de género [/http://www. equalitaca.org/ nuevaitaca / documentosforo/ soniaparella.pdf〉].

- (2000): "El trasvase de desigualdad de clase y etnia entre mujeres: los servicios de proximidad", Papers, no 60 , págs. $275-289$.

PÉREZ OROZCO, A.; y LÓPEZ GIL, S. (2011): Desigualdades a flor de piel: cadenas globales de cuidados. Concreciones en el empleo de hogar y políticas públicas, Madrid, ONU Mujeres.

PÉREZ SÁNCHEZ, C.; CARDÚS, S.; y MORRAL, S. (2002): Propostes d'intervenció per a la conciliació d'horaris familiars, escolars i laborals, Barcelona, Generalitat de Catalunya, Departament de Benestar Social.

RIVAS, A. M. (2006): “El empleo o la vida: perder el empleo para conservar la vida o renunciar a la vida para conservar el empleo ¿de qué conciliación hablamos?", AIBR. Revista de Antropología lberoamericana, vol. 1, $\mathrm{n}-3$, págs. 367-374.

ROYO, R. (dir.) (2012): Corresponsabilidad, valores y género, Vitoria-Gasteiz: Emakunde.

- (2011): Maternidad, paternidad y conciliación en la CAE: ¿es el trabajo familiar un trabajo de mujeres?, Bilbao, Universidad de Deusto [en colaboración con Emakunde].

SÁNCHEZ MUÑOZ, C. (2001): "Genealogía de la vindicación”, en BELTRÁN, C.; y MAQUIEIRA, V. (eds.), Feminismos. Debates teóricos contemporáneos, Madrid, Alianza, págs. 17-73.

SILVESTRE, M. (2007): "La conciliación familiar en la Ley 4/2005 de 18 de febrero para la Igualdad de Hombres y Mujeres", en Actas VII Congreso Vasco de Sociología y Ciencia Política, Bilbao, Asociación Vasca de Sociología y Ciencia Política.

TOBÍO, C. (2005): Madres que trabajan. Dilemas y estrategias, Madrid, Cátedra.

- (1998): “Roles de género y la relación familia-empleo”, Asparkía. Investigació Feminista, nํㅜ págs. 21-44.

TORNS, T. (2005): "De la imposible conciliación a los permanentes malos arreglos", Cuadernos de Relaciones Laborales, 23, ํㅡㅅㅡ. págs. 15-33.

TORNS, T.; BORRÀS, V.; y CARRASQUER, P. (2003): “La conciliación de la vida laboral y familiar: ¿un horizonte posible?", Sociología del Trabajo, nํํㄴ 50 , págs. 111-137.

VALIENTE, C. (2005): "The changing roles of men in families in Spain", en THRELFALL, M.; COUSINS, C.; y VALIENTE C., Gendering Spanish Democracy, Londres, Routledge, págs. 187-203. 